\title{
FRENDY: A new nuclear data processing system being developed at JAEA
}

\author{
Kenichi Tada ${ }^{a}$, Yasunobu Nagaya, Satoshi Kunieda, Kenya Suyama, and Tokio Fukahori \\ Japan Atomic Energy Agency, Tokai, Japan
}

\begin{abstract}
JAEA has provided an evaluated nuclear data library JENDL and nuclear application codes such as MARBLE, SRAC, MVP and PHITS. These domestic codes have been widely used in many universities and industrial companies in Japan. However, we sometimes find problems in imported processing systems and need to revise them when the new JENDL is released. To overcome such problems and immediately process the nuclear data when it is released, JAEA started developing a new nuclear data processing system, FRENDY in 2013. This paper describes the outline of the development of FRENDY and both its capabilities and performances by the analyses of criticality experiments. The verification results indicate that FRENDY properly generates ACE files.
\end{abstract}

\section{Introduction}

Japan Atomic Energy Agency (JAEA) provides an evaluated nuclear data library JENDL [1] and nuclear application codes, e.g., a versatile reactor analysis code system MARBLE2 [2], a comprehensive neutronics calculation code system SRAC [3,4], a general purpose Monte Carlo code MVP [5], and a particle and heavy-ion transport code system PHITS [6].

Currently, the nuclear data libraries for these domestic codes are processed with combined systems of their original modules and other modules of NJOY [7,8] and PREPRO [9]. When the new JENDL was released, these processing systems sometimes have problems and files were needed to be modified. Furthermore, since NJOY and PREPRO are imported systems, JAEA cannot release their nuclear data processing system. Therefore, the nuclear application code users cannot process the nuclear data library by themselves. For these reasons, when the new JENDL is released, the users cannot apply the new nuclear data library to nuclear application codes immediately and they have to wait until JAEA releases new cross section libraries.

Recently, the introduction of the Generalized Nuclear Data (GND) utilizing the XML format has been considered [10]. Since the XML format is completely different from the current ENDF-6 format [11], the current widely used nuclear data processing systems cannot treat the GND format without extensive modification.

To overcome such problems, JAEA started to develop a new nuclear data processing system FRENDY (FRom Evaluated Nuclear Data libralY to any application) in 2013. To develop FRENDY in JAEA by concentrating the appropriate knowledge, a development team was established in Nuclear Science and Engineering Center

a e-mail: tada.kenichi@jaea.go.jp of JAEA [12], having members of the nuclear data evaluators and specialists on neutronics calculations. The development team and other JAEA colleagues enhanced understanding of the methods of nuclear data processing systems and it contributes considerably to the development of FRENDY.

In order to obtain the needs and feedback from potential users of FRENDY in Japan, the status of development has been reported in the nuclear data processing working group in the JENDL committee.

The outline of the development of FRENDY, its capabilities and performances are presented in this paper. For the verification of FRENDY, comparisons are made for the processed results of FRENDY and NJOY99 and the $\mathrm{k}_{\mathrm{eff}}$ values using the ACE file with processed libraries.

\section{Overview of FRENDY}

FRENDY is designed with an emphasis on maintainability, modularity, portability and flexibility. To achieve these requirements, FRENDY is written in an object-oriented language $\mathrm{C}++$. Since all classes in FRENDY are encapsulated, maintainability and modularity are greatly improved from current nuclear data processing systems written in FORTRAN77. Furthermore, to enhance portability and flexibility, each class is carefully designed to minimize the function and to maintain the independence of each module.

Modules of FRENDY such as the resonance reconstruction and Doppler broadening are also encapsulated. These modules can be used with other calculation codes by adding only a few lines. Each module of FRENDY can be easily revised and extended for the future needs.

To assure the quality and reliability, the verification of each class and function are also considered. The Boost Test library is used to verify each class and function. For the

(C) The Authors, published by EDP Sciences. This is an Open Access article distributed under the terms of the Creative Commons Attribution License 4.0 (http://creativecommons.org/licenses/by/4.0/). 


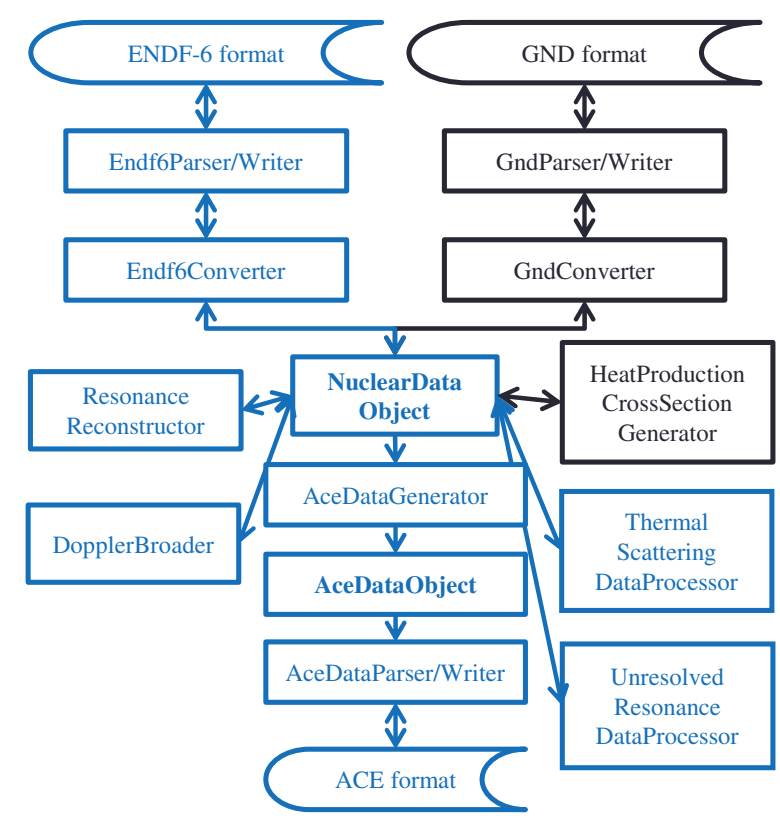

Figure 1. The structure of FRENDY.

source code management, the version control system Git [13] is used.

The structure of FRENDY is shown in Fig. 1. The blue-colored modules have been already implemented, while the black-colored ones have not been implemented yet. Though the current version of FRENDY cannot treat the general R-matrix format, the module of the R-matrix code AMUR [14] will be imported to process it in the near future. As shown in Fig. 1, FRENDY converts a set of nuclear data in ENDF format to an object of the "NuclearDataObject" class which has its own data structure. Using the NuclerDataObject class, FRENDY can be easily extended to support a new nuclear data format. Though the current version of FRENDY supports the traditional ENDF-6 format only, FRENDY can address the GND or the other nuclear data formats if new parser, writer and converter classes are implemented.

\section{Verification of FRENDY}

To verify FRENDY, the processed results are compared to those of NJOY99.393 [7]. All nuclei and materials prepared in JENDL-4.0 and JENDL-3.3 are used to verify FRENDY. In this paper, the verification of the Doppler broadening and processing the thermal scattering law data is described.

The fractional tolerance used for linearizing in resonance reconstruction, Doppler broadening and processing the thermal scattering law data is $0.01 \%$. Generally, the tolerance value is $0.1 \%$ to generate the ACE files. In this study, to compare the cross section values which are obtained by FRENDY and NJOY99 in more detail, such a strict tolerance value is used. The other calculation conditions of these calculations are identical.

Only the verification of Doppler broadening and processing the thermal scattering law data is shown in the following; other modules to calculate probability tables and generate ACE data files have been already verified.

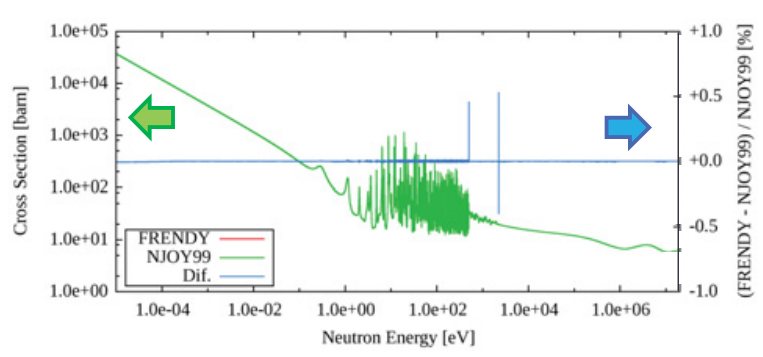

Figure 2. Comparison of the total cross section for ${ }^{235} \mathrm{U}$ from JENDL-4.0 at $293.6 \mathrm{~K}$.

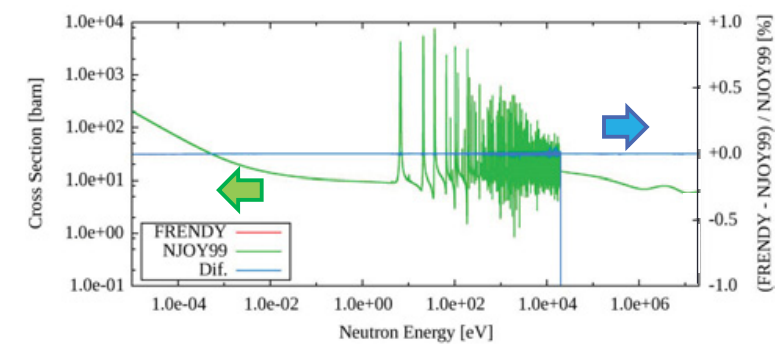

Figure 3. Comparison of the total cross section for ${ }^{238} \mathrm{U}$ from JENDL-4.0 at $1200 \mathrm{~K}$.

\subsection{Doppler broadening}

Comparisons are made for the total cross sections of ${ }^{235} \mathrm{U}$ and ${ }^{238} \mathrm{U}$ from JENDL-4.0 [1]. Figures 2 and 3 show the Doppler broadened cross sections at $293.6 \mathrm{~K}$ and $1200 \mathrm{~K}$, respectively, to verify the Doppler broadening process.

To calculate the Doppler broadened cross sections, resonance reconstruction is required. The resonance reconstruction is also processed by FRENDY and NJOY99. So, the difference of the cross section in Figs. 2 and 3 include both the difference of resonance reconstruction and of Doppler broadening.

As shown in Figs. 2 and 3, the difference is only found in the boundary of the resolved and unresolved resonance regions. Since the boundary of the resolved and unresolved resonance regions is discontinuous, such difference is observed. However, as show in Figs. 2 and 3 , the difference at the discontinuity region is not so large. Therefore, this difference will not affect neutronics calculations in general.

The other reaction types, e.g., scattering, radiative capture and fission, and nuclei prepared in JENDL-4.0 and JENDL-3.3 also exhibit similar difference. These results indicate that the resonance reconstruction and the Doppler broadening process in FRENDY are appropriate.

\subsection{Thermal scattering law data}

Comparisons of the coherent elastic and inelastic scattering cross sections for ${ }^{1} \mathrm{H}$ in liquid $\mathrm{H}_{2} \mathrm{O}$ from JENDL-4.0 are shown in Figs. 4 and 5.

The difference is found only at the discontinuous point in the coherent elastic cross section. Similar differences can be also found for the other materials prepared in JENDL-4.0 and JENDL-3.3. These results indicate that the processing the thermal scattering law data in FRENDY is appropriate. 


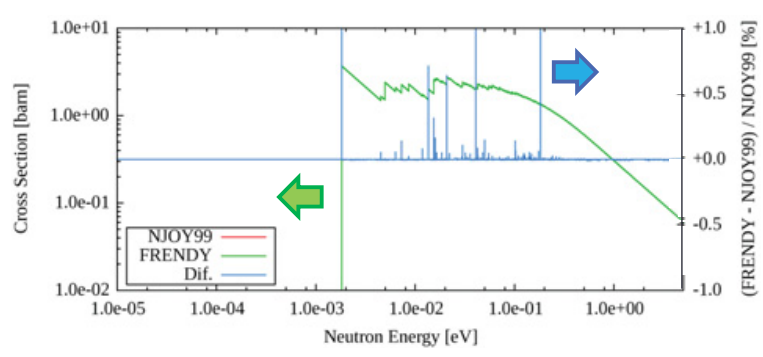

Figure 4. Comparison of the coherent elastic scattering cross section for ${ }^{1} \mathrm{H}$ in a liquid light water from JENDL-4.0 at $296 \mathrm{~K}$.

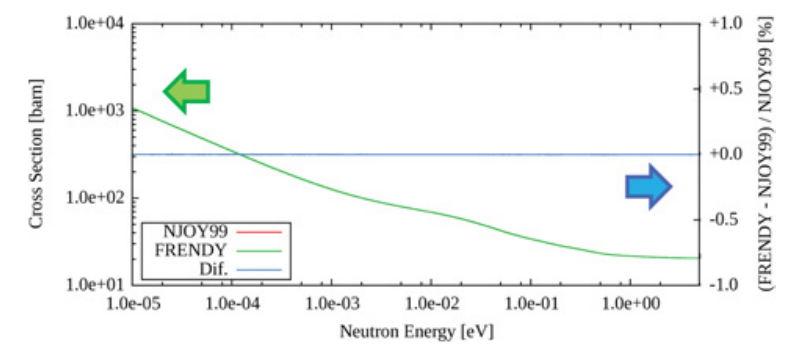

Figure 5. Comparison of the inelastic scattering cross section for ${ }^{1} \mathrm{H}$ in a liquid light water from JENDL-4.0 at $296 \mathrm{~K}$.

Table 1. The total processing time and the ratio to generate the ACE file processed by FRENDY and NJOY99.

\begin{tabular}{|l|c|c|c|}
\hline & FRENDY [s] & NJOY99 [s] & FRENDY/NJOY99 \\
\hline${ }^{16} \mathrm{O}$ & 1.83 & 0.70 & 2.61 \\
\hline${ }^{56} \mathrm{Fe}$ & 18.00 & 9.10 & 1.98 \\
\hline${ }^{235} \mathrm{U}$ & 944.28 & 435.80 & 2.17 \\
\hline${ }^{238} \mathrm{U}$ & 581.94 & 437.40 & 1.33 \\
\hline${ }^{1} \mathrm{H}$ in $\mathrm{H}_{2} \mathrm{O}$ & 49.03 & 15.30 & 3.20 \\
\hline graphite & 36.96 & 9.50 & 3.89 \\
\hline
\end{tabular}

\subsection{Comparison of the processing time}

To estimate the efficiency of process by FRENDY, the total processing time to generate the ACE files is compared.

Table 1 shows the total processing time to generate the ACE files in each nucleus and material. Note that the tolerance value used for generating the ACE files is $0.1 \%$. The temperature of each nucleus and material is set to $296 \mathrm{~K}$. The other calculation conditions to make ACE files are identical.

As shown in Table 1, the processing time of FRENDY is 2 or 3 times longer than that of NJOY99. This processing time difference is also observed in other nuclei and materials prepared in JENDL-4.0 and JENDL-3.3.

The cause of the difference is mainly the difference of the programming language and using dynamic arrays. NJOY99 is written in FORTRAN77. Generally speaking, the calculation time of the FORTRAN77 program is faster than that of the $\mathrm{C}++$ program. Furthermore, FRENDY uses the dynamic arrays to improve maintainability and readability. The dynamic array requires longer processing time comparing to the static arrays used in NJOY99.

Since the computer performance is constantly improving and nuclear data library is not frequently processed, current processing time with FRENDY is acceptable.

\section{Comparisons of integral experiments}

To verify FRENDY comprehensively, the calculation results of MCNP5 [15] using the ACE files from JENDL4.0 processed by FRENDY are compared to those by NJOY99.393. The tolerance value to generate the ACE files is $0.1 \%$. The other calculation conditions are identical.

To compare the neutronics calculations, a few dozen integral experiments in the ICSBEP handbook [16] are used. The MCNP sample input files in the ICSBEP hand book are used for comparison. Only the nuclide identifiers in the sample input files are modified to use the ACE files processed by FRENDY and NJOY99.

Many MCNP sample input files in the ICSBEP hand book are not intended to be used directly for validation. In this study, these sample input files are not used to compare the experimental results. These sample input files are only used to compare the calculation results, i.e., the $\mathrm{k}_{\text {eff }}$ value, using the ACE files processed by FRENDY and NJOY99. Even if the calculation results are different from the experimental results, there is no impact on the verification of FRENDY. For these reasons, using the original MCNP sample input files in the ICSBEP hand book is appropriate for the verification of FRENDY.

Table 2 shows the benchmark problems used in this comparison, the $\mathrm{k}_{\mathrm{eff}}$ values and the relative differences. As shown in Table 2, to estimate the difference of $\mathrm{k}_{\mathrm{eff}}$ in soft and hard neutron spectra, both thermal and fast spectrum experimental data are selected. The impact of the difference of the major fissile materials, i.e., ${ }^{235} \mathrm{U},{ }^{239} \mathrm{Pu}$ and ${ }^{233} \mathrm{U}$, is also estimated.

As shown in Table 2, the $\mathrm{k}_{\mathrm{eff}}$ values of FRENDY are similar to those of NJOY99. Furthermore, the relative differences are not so varied with the neutron spectrum and the major fissile materials.

These results indicate that FRENDY properly generates the ACE files.

\section{Conclusions and future works}

JAEA has started to develop a new nuclear data processing system FRENDY in order to immediately process the nuclear data library when a new JENDL is released. FRENDY is designed to support the new nuclear data format, e.g., the GND format. FRENDY is developed under careful consideration for maintainability, modularity, portability and flexibility.

To verify the process results, the process results of FRENDY are compared to those of NJOY99. The processing results indicate that the difference is very small.

To compare the ACE files of FRENDY and NJOY99, a few dozen integral experiments in the ICSBEP handbook are used for comparison of the neutronics calculations. The $\mathrm{k}_{\mathrm{eff}}$ values of FRENDY are similar to those of NJOY99. These results indicate that FRENDY properly generates the ACE files.

Because FRENDY has been developed to solve the numerous problems of the current nuclear data processing systems, the methods adopted in FRENDY are similar to those in NJOY99. The modification of the processing methods and implementation of the original functions will be investigated based on this experience. 
Table 2. The $\mathrm{k}_{\mathrm{eff}}$ values and the relative differences for various integral experimental benchmark problems using ACE files processed by FRENDY and NJOY99.

\begin{tabular}{|c|c|c|c|c|c|c|}
\hline \multirow[b]{2}{*}{ Bench mark } & \multirow[b]{2}{*}{ Case No } & \multicolumn{2}{|r|}{ FRENDY } & \multicolumn{2}{|r|}{ NJOY99 } & \multirow[b]{2}{*}{ (FRENDY-NJOY99)/NJOY99 } \\
\hline & & $\mathrm{k}_{\mathrm{eff}}$ & standard deviation & $\mathrm{k}_{\mathrm{eff}}$ & standard deviation & \\
\hline \multirow{5}{*}{ HMF7 } & 1 & 0.99088 & $0.01 \%$ & 0.99094 & $0.01 \%$ & $-0.01 \%$ \\
\hline & 2 & 0.99644 & $0.01 \%$ & 0.99639 & $0.01 \%$ & $0.01 \%$ \\
\hline & 3 & 0.99792 & $0.02 \%$ & 0.99803 & $0.02 \%$ & $-0.01 \%$ \\
\hline & 4 & 0.99656 & $0.02 \%$ & 0.99658 & $0.02 \%$ & $0.00 \%$ \\
\hline & 5 & 0.99892 & $0.02 \%$ & 0.99878 & $0.02 \%$ & $0.01 \%$ \\
\hline \multirow{4}{*}{ ICI1 } & 18 & 0.97242 & $0.01 \%$ & 0.97227 & $0.01 \%$ & $0.02 \%$ \\
\hline & 19 & 0.98134 & $0.01 \%$ & 0.98129 & $0.01 \%$ & $0.01 \%$ \\
\hline & 20 & 1.02365 & $0.02 \%$ & 1.02365 & $0.02 \%$ & $0.00 \%$ \\
\hline & 21 & 0.94048 & $0.02 \%$ & 0.94060 & $0.02 \%$ & $-0.01 \%$ \\
\hline \multirow{5}{*}{ LCT6 } & 1 & 0.99867 & $0.02 \%$ & 0.99834 & $0.02 \%$ & $0.03 \%$ \\
\hline & 2 & 0.99916 & $0.02 \%$ & 0.99943 & $0.02 \%$ & $-0.03 \%$ \\
\hline & 3 & 0.99880 & $0.02 \%$ & 0.99882 & $0.02 \%$ & $0.00 \%$ \\
\hline & 4 & 0.99839 & $0.02 \%$ & 0.99872 & $0.02 \%$ & $-0.03 \%$ \\
\hline & 5 & 0.99891 & $0.02 \%$ & 0.99865 & $0.02 \%$ & $0.03 \%$ \\
\hline \multirow{5}{*}{ MCT12 } & 1 & 0.97329 & $0.02 \%$ & 0.97341 & $0.02 \%$ & $-0.01 \%$ \\
\hline & 2 & 0.97458 & $0.02 \%$ & 0.97445 & $0.02 \%$ & $0.01 \%$ \\
\hline & 3 & 0.97217 & $0.02 \%$ & 0.97193 & $0.02 \%$ & $0.02 \%$ \\
\hline & 4 & 0.97589 & $0.02 \%$ & 0.97594 & $0.02 \%$ & $-0.01 \%$ \\
\hline & 5 & 0.97426 & $0.02 \%$ & 0.97444 & $0.02 \%$ & $-0.02 \%$ \\
\hline \multirow{4}{*}{ PMF1 } & 1 & 0.99905 & $0.01 \%$ & 0.99887 & $0.01 \%$ & $0.02 \%$ \\
\hline & 2 & 0.99943 & $0.01 \%$ & 0.99965 & $0.01 \%$ & $-0.02 \%$ \\
\hline & 3 & 0.99928 & $0.01 \%$ & 0.99918 & $0.01 \%$ & $0.01 \%$ \\
\hline & 4 & 1.00019 & $0.01 \%$ & 1.00011 & $0.01 \%$ & $0.01 \%$ \\
\hline \multirow{5}{*}{ UCT1 } & 1 & 0.98448 & $0.02 \%$ & 0.98428 & $0.02 \%$ & $0.02 \%$ \\
\hline & 2 & 0.98389 & $0.02 \%$ & 0.98420 & $0.02 \%$ & $-0.03 \%$ \\
\hline & 3 & 0.98511 & $0.02 \%$ & 0.98528 & $0.02 \%$ & $-0.02 \%$ \\
\hline & 4 & 0.97634 & $0.02 \%$ & 0.97655 & $0.02 \%$ & $-0.02 \%$ \\
\hline & 5 & 0.97952 & $0.02 \%$ & 0.97900 & $0.02 \%$ & $0.05 \%$ \\
\hline
\end{tabular}

The other modules, e.g., generation of cross section for multi group neutronics calculation codes, processing the covariance data and calculation of KERMA factor and DPA cross section, will be also implemented in the future.

The authors wish to thank Prof. J. Katakura, Prof. G. Chiba, Prof. T. Endo, Dr. T. Hino, Dr. K. Yamaji, Dr. O. Iwamoto, Dr. T. Kugo, Dr. C. Konno, Mr. K. Okumura, Mr. M. Tokashiki, Mr. R. Kimura, Mr. T. Sakai and Mr. D. Iwahashi who are the members of the nuclear data processing working group of the JENDL committee for their expert advices and encouragements. The authors also wish to thank JAEA colleagues for their expert advices and suggestions. Their fine advices and suggestions are greatly helpful to develop and improve FRENDY.

\section{References}

[1] K. Shibata, O. Iwamoto, et al., "JENDL-4.0: A New Library for Nuclear Science and Engineering," J. Nucl. Sci. Technol. 48, 1-30 (2011)

[2] K. Yokoyama, T. Hazama, et al, "Development of comprehensive and versatile framework for reactor analysis, MARBLE," Ann. Nucl. Energy 66, 51-60 (2014)

[3] K. Okumura, T. Kugo, et al, "SRAC2006: A Comprehensive Neutronics Calculation Code System," JAEA-Data/Code 2007-004 (2007)

[4] K. Okumura, "MOSRA-SRAC; Lattice calculation module of the modular code system for nuclear reactor analyses MOSRA," JAEA-Data/Code, 2015-015 (2015)

[5] Y. Nagaya, K. Okumura, et al, "MVP/GMVP II: General Purpose Monte Carlo Codes for Neutron and Photon Transport Calculations based on Continuous Energy and Multigroup Methods," JAERI-1348 (2004)

[6] H. Iwase, K. Nitta, T. Nakamura, "Development of general-purpose particle and heavy ion transport Monte Carlo code," J. Nucl. Sci. Technol. 39, 1142-1151 (2002)

[7] R. E. MacFarlane, D. W. Muir, "The NJOY Nuclear Data Processing System Version 91," LA-12740-M (1994)

[8] D. W. Muir, R. M. Boicourt, A. C. Kahler, "The NJOY Nuclear Data Processing System, Version 2012," LA-UR-12-27079 (2012)

[9] D. E. Cullen, "PREPRO 2012, 2012 ENDF/B Preprocessing Codes (ENDF/B-VII Tested)," IAEANDS-39, Rev.15 (2012)

[10] C. M. Mattoon, B. R. Beck, et al, "Generalized Nuclear Data: A New Structure (with Supporting Infrastructure) for Handling Nuclear Data," Nuclear Data Sheets 113, 3145-3171 (2012)

[11] A. Trkov, M. Herman, D. A. Brown, "ENDF-6 Formats Manual," BNL-90365 2009 Rev. 2 (2011)

[12] K. Suyama, "Direction of alternative code of NJOY," research for reactor physics 66, 11-14 (2014) [in Japanese] 
[13] S. Chacon, B. Straub, "Pro Git 2nd Edition," Apress, ISBN-13: 978-1484200773 (2014)

[14] S. Kunieda, "Status of the R-matrix code AMUR toward a consistent cross-sections evaluation and covariance analysis for the light nulei," Proc. ND 2016, Sep. 11-16, 2016, Bruges, Belgium (2016)
[15] X-5 Monte Carlo Team, "MCNP - A General Monte Carlo N-Particle Transport Code, Version 5," LA-UR-03-1987 (2003)

[16] OECD/NEA, "International Handbook of Evaluated Criticality Safety Benchmark Experiments," NEA/NSC/DOC(95)03 